\title{
Recurrencia de infección por COVID-19 en población general inmunizada
}

(c) (i) (a) (2)

Recurrence of COVID-19 infection in inmunitized Health personnel

Joamely Evelin Bermeo González. ${ }^{1}$, Tamara Alejandra Salamea Montenegro. ${ }^{2}$, María Emilia Delgado Larrea. ${ }^{3}$, Stefanía Esther Montalván Zambrano. ${ }^{4}$ \& Jaime Santiago Sánchez Solórzano. ${ }^{5}$

DOI: https://doi.org/10.33262/anatomiadigital.v4i4.1941

\begin{abstract}
.
Resumen.

The recurrence of COVID-19 is not very La recurrencia de la COVID-19, no está well determined at present, the muy bien determinada en la actualidad, probabilities of viral reactivation, new infection or infection by a new strain are las probabilidades de reactivación viral, nueva infección o infección por una those studied now. The objective was to identify the recurrence of SARS CoV-2 infection, blood group and type of nueva cepa son las estudiadas al momento. El objetivo fue identificar la recurrencia de infección por SARS CoVvaccine in patients immunized for COVID-19. A descriptive study was 2, grupo sanguíneo y tipo de vacuna en pacientes inmunizados para la COVIDcarried out with the results of surveys 19. Se realizó un estudio descriptivo con directed at the general population of a resultados de encuestas dirigidas a

\footnotetext{
${ }^{1}$ Interhospital, Auditoría Médica, Guayaquil, Ecuador. joamelyta12@hotmail.com

${ }^{2}$ Cruz Vital, S.A, Cuenca, Ecuador. tamarapa_35@ @otmail.com

3 Centro Médico Semedic Urdesa, Consulta Externa. Guayaquil, Ecuador. maemilia_delgado@hotmail.com

4 Hospital IESS Machala, Hospitalización Clínica, Machala, Ecuador. estefania.montalvanzb@hotmail.com

5 Hospital General Monte Sinaí, Unidad de Cuidados Intensivos, Guayaquil, Ecuador. jaimes.sanchezs@hotmail.com
} 
group of social networks. A database was created in Microsoft Excel 2016, it was processed with SPSS 25, frequencies and percentages of qualitative and quantitative variables were calculated. It was found that the recurrence of COVID-19 was $6.30 \%$ of the sample studied, with $3.6 \%$ frequency in people with blood type $\mathrm{O} \mathrm{Rh}+$. The protocolization of care for patients with COVID-19 for immunized patients is important to identify in a timely manner the clinical, demographic and complementary laboratory characteristics to identify recurrences of infection by SARS CoV-2.

Keywords: SARS CoV-2, COVID-19, recurrence, infection. población general de un grupo de redes sociales. Se creó una base de datos en Microsoft Excel 2016, se procesó con SPSS 25, se calcularon frecuencias y porcentajes de variables cualitativas y cuantitativas. Se encontró que la recurrencia de la COVID-19 fue del $6,30 \%$ de la muestra estudiada, con el $3,6 \%$ de frecuencia en personas con tipo de sangre $\mathrm{O} \mathrm{Rh}+$. Es importante la protocolización de atención a pacientes con COVID-19 para pacientes inmunizados con la finalidad de identificar de forma oportuna las características clínicas, demográficas y complementarias laboratoriales con la finalidad de identificar las recurrencias de infección por SARS CoV-2.

Palabras claves: SARS CoV-2, COVID-19, recurrencia, infección.

\section{Introducción}

En la ciudad de Wuhan (China), a finales del año 2019 inició un brote de casos por neumonía grave que hasta la actualidad no sabemos el origen exacto de la misma. De acuerdo con estudios epidemiológicos esta enfermedad se pronosticó que seguiría avanzando y llegaríamos a una pandemia. La agresividad de esta enfermedad causada por el virus del SARS CoV-2, inicialmente mostró una afinidad con las personas adultas entre 30 a 79 años, con una letalidad del 2.3\% (Díaz-Castrillón, 2020; Guanche \& Barbán, 2021; Salao, 2020).

En la literatura científica se encuentra reportada la reinfección con grados bajos de ocurrencia, debido a que en la actualidad aún se siguen realizando y determinando estudios de características para su identificación clara y precisa, lo más probable es que podría ser una reactivación viral por un contagio previo, infección por la misma cepa del virus o infección por una cepa diferente (Fatehi, 2021).

Existen estudios los cuales catalogan a una reinfección a la detección de ARN viral cuando previamente se ha presentado dos muestras de hisopado nasofaríngeo con secuenciación negativa. En la actualidad se desconoce además el tiempo de duración y/o protección de la respuesta inmunitaria secundario a una infección por SARS CoV-2. 
Los estudios genómicos son determinantes a la hora de poder definir a una reactivación o reinfección viral (Tillett, 2020; Viera, 2020).

El conocimiento de la fisiopatología, así como del comportamiento inmunológico post infección por SARS CoV-2 o post inmunización son necesarios para poder determinar si las tasas de nuevos contagios se deben a reinfección o reactivación de la COVID-19, con la finalidad de protocolarizar este proceso según el comportamiento clínico y laboratorial en beneficio de toda la población y contribuir con la prevención de la enfermedad, promoción de la salud y posterior rehabilitación post COVID-19 (Tillett, 2020).

Las conclusiones que se pueden obtener de todos los reportes de casos son muy limitadas, actualmente es necesario realizar estudios multicéntricas con un grupo más grande de pacientes con características clínicas de infección y probable reinfección para definir con mayor exactitud la definición de este término (Overbaugh, 2020), por los antecedentes expuestos, nosotros catalogamos a recurrencia la presentación de nuestro estudio, mas no como reinfección.

El objetivo del presente estudio fue identificar la frecuencia de recurrencia de infección por SARS CoV-2 en personas previamente inmunizadas y lograr determinar el grupo de personas con mayor afección por el grupo sanguíneo, así como también demostrar el porcentaje de la población con los diferentes tipos de vacunas.

\section{Metodología}

Se realizó un estudio descriptivo, transversal. Se realizó una encuesta con preguntas dirigidas a la población general de nuestro país Ecuador.

El período de las encuestas realizadas fue entre la primera y segunda semana del mes de noviembre del 2021. El formulario se socializó a 500 personas de un grupo de anuncios de redes sociales. Se incluyó personas mayores de 18 años con nacionalidad ecuatoriana, se indagó sobre edad, género, etnia, provincia de residencia, grupo sanguíneo, antecedentes de infección por SARS CoV-2 e inmunización para la COVID19.

Las variables solicitadas fueron recolectadas en Microsoft Excel 2016 y los datos se procesaron con el programa SPSS 25, con cálculo de variables cualitativas y cuantitativas.

Para utilizar la información recolectada se solicitó inicialmente responder al consentimiento informado para la publicación de los resultados como apoyo a la comunidad científica. El presente estudio cumple con los principios éticos de Helsinki.

\section{Resultados y discusión}

Todos los pacientes $(n=111)$ aceptaron el consentimiento informado de utilizar la información solicitada con fines académico - científicos. 
El género que tuvo más respuestas al formulario fue el femenino, con una media de edad de 26,89 años, la etnia mestiza, encuestados de la provincia residencial en Pichincha con grupo sanguíneo tipo $\mathrm{O} R \mathrm{Rh}+$ fueron los más frecuentes, como se muestra en la tabla1.

\section{Tabla 1}

Características sociodemográficas de la población inmunizada

\begin{tabular}{|c|c|c|c|}
\hline \multicolumn{2}{|c|}{ Variables } & No & $\%$ \\
\hline \multirow[t]{2}{*}{ Género } & Masculino & 52 & $46,85 \%$ \\
\hline & Femenino & 59 & $53,15 \%$ \\
\hline \multirow[t]{3}{*}{ Edad } & Media (DE) 26,89 & & \\
\hline & Mediana (RIQ) 23 & & \\
\hline & Edad min-máx. (1 & & \\
\hline \multirow[t]{4}{*}{ Etnia } & Mestizo & 98 & $88,29 \%$ \\
\hline & Blanco & 9 & $8,11 \%$ \\
\hline & Montubio & 3 & $2,70 \%$ \\
\hline & Indígena & 1 & $0,90 \%$ \\
\hline \multirow[t]{10}{*}{ Provincia residencia } & Pichincha & 73 & $65,77 \%$ \\
\hline & Cotopaxi & 10 & $9,01 \%$ \\
\hline & Manabí & 10 & $9,01 \%$ \\
\hline & Imbabura & 8 & $7,21 \%$ \\
\hline & Guayas & 5 & $4,50 \%$ \\
\hline & Azuay & 1 & $0,90 \%$ \\
\hline & Carchi & 1 & $0,90 \%$ \\
\hline & El Oro & 1 & $0,90 \%$ \\
\hline & Morona Santiago & 1 & $0,90 \%$ \\
\hline & Orellana & 1 & $0,90 \%$ \\
\hline \multirow[t]{7}{*}{ Tipo de sangre } & $\mathrm{O} \mathrm{Rh}+$ & 70 & $63,06 \%$ \\
\hline & A Rh+ & 16 & $14,41 \%$ \\
\hline & O Rh- & 9 & $8,11 \%$ \\
\hline & B Rh+ & 6 & $5,41 \%$ \\
\hline & A Rh- & 4 & $3,60 \%$ \\
\hline & $\mathrm{AB} \mathrm{Rh}+$ & 3 & $2,70 \%$ \\
\hline & Desconoce & 3 & $2,70 \%$ \\
\hline
\end{tabular}

Elaborado por: Los autores

Del total de los encuestados, aproximadamente la tercera parte refirió haber presentado infección por SARS CoV-2 con mayor frecuencia en el año 2020 y 2021. Los meses con mayor número de contagios fueron aquellos en los cuales existió un antecedente de feriado y/o vacaciones en la población ecuatoriana, como se muestra en la tabla 2.

Por el inicio del esquema de inmunización oportuno durante este año, probablemente los porcentajes de frecuencia en cuanto a gravedad por la COVID-19 han disminuido, se evidencia que el 23,42\% fueron tratados ambulatoriamente. Toda la población de estudio fue inmunizada, con una media de dosis administrada de 2 vacunas. En la primera y segunda dosis de inmunización predominó la vacuna Pfizer y en la tercera dosis Aztrazeneca (tabla 2). 


\section{Tabla 2}

Características de recurrencia e inmunización de la población

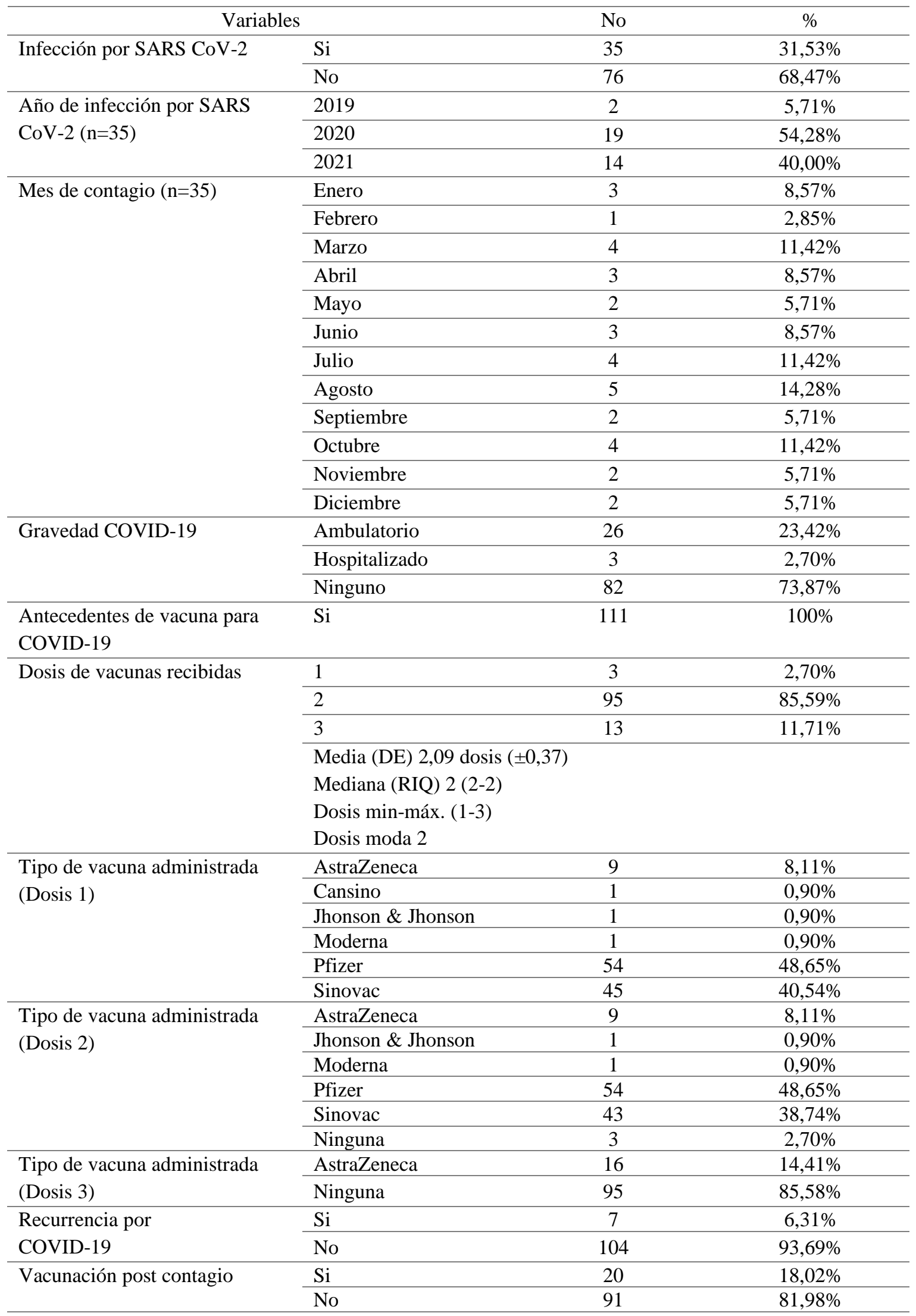

Elaborado por: Los autores 
La recurrencia de la COVID-19 fue del 6,30\% (n=7) de la muestra estudiada, detectada clínicamente y con algún tipo de estudio diagnóstico para COVID-19. En nuestra población estudiada, de acuerdo con el tipo de sangre, las personas con recurrencia de la enfermedad COVID-19 fueron las de tipo O Rh+ 57,14\% (n=4), O Rh- 28,57\% (n=2) y $\mathrm{B} \mathrm{Rh}+14,28 \%(\mathrm{n}=1)$. Algo interesante es que existió un porcentaje considerable $18,02 \%$ de personas que fueron vacunadas luego de la infección por SARS CoV-2, existiendo como hipótesis el inicio del programa de inmunización o la importancia de la vacuna luego de la infección experimentada.

De acuerdo con la revisión de la literatura, la reinfección no está muy bien determinada y/o establecida, generalmente la información obtenida y reportes realizados son dirigidos a la recurrencia de la enfermedad (Fatehi, 2021), terminología tomada como la presentación de signos o síntomas por una segunda o tercera ocasión, sin ser necesariamente confirmado el diagnóstico mediante la realización del hisopado nasofaríngeo.

Según estudios, existe la propuesta de considerar a la recurrencia por COVID-19 si la segunda infección ocurrió 21 días posterior a un período libre de síntomas, excluyendo las etiologías alternativas (Gousseff, 2020).

En un estudio de Wuhan, China, el 9\% de pacientes $(n=5 / 55)$ tuvo una recurrencia de la enfermedad. Tanto los pacientes asintomáticos como los mínimamente sintomáticos tenían el potencial de reactivarse. Según el estudio la recurrencia de la infección por SARS CoV-2 fue confirmada mediante el secuenciamiento de la PCR del hisopado orofaríngeo. Las características clínicas presentadas fueron fiebre, tos, odinofagia y fatiga. En el estudio se indica que en complementarios laboratoriales, la linfopenia y la neutrofilia predominaron en los pacientes sintomáticos considerados como reactivados (Ye et al., 2020).

Wang to: Existen reportes considerados como reinfección humana por SARS CoV-2, como el primer caso divulgado en Hong Kong en el mes de agosto del 2020, mediante la secuenciación genética de muestras recolectadas mediante hisopado nasal del mismo paciente con un lapso de 142 días. Existió evidencia de que los genomas virales pertenecieron a diferentes linajes, uno de los cuales fue más incidente entre marzo y abril de 2020, mientras que el otro se acerca a las cepas encontradas en la actualidad (To, 2021).

Análisis de epidemiológicos, clínicos, serológicos y genómicos según reportes de reinfección en pacientes, sugieren que el virus SARS CoV-2 podría continuar propagándose a pesar de los niveles de inmunización (To, 2021).

De acuerdo con nuestros reportes, se confirma la gravedad de la enfermedad COVID19. Con este antecedente es importante socializar a la comunidad en general la necesidad de continuar con las medidas de prevención/protección como higiene adecuada de manos, uso de alcohol gel, distanciamiento social, y uso de mascarilla evitando así las probables recurrencias. Según reportes de recurrencias y/o reinfecciones 
no existen datos específicos sobre la relación entre el tipo de grupo sanguíneo y este fenómeno de sintomatología a repetición (Tillett, 2020; Overbaugh, 2020).

En la literatura se ha encontrado reportes de series de casos clínicos, con tasas de reinfección entre el 3 al 31\%, es desconocido actualmente el porcentaje de protección de la respuesta inmunitaria frente a nuevas infecciones por el virus SARS CoV-2, se ha sugerido que la presencia de inmunoglobulina $\mathrm{M}$ en los procesos agudos de la enfermedad es de utilidad diagnóstica, pero su ausencia no la excluye de una reinfección y/o recurrencia (Romera, 2021).

\section{Conclusiones}

- La recurrencia de la infección por SARS CoV-2, considerando la presencia de sintomatología presente y confirmada mediante métodos diagnósticos, fue considerable en pacientes inmunizados de la población general. Es importante protocolizar y lograr determinar las características clínicas y demográficas (signos/síntomas/grupo sanguíneo, por ejemplo), así como incluir la secuenciación genómica para identificar las verdaderas reinfecciones producidas o reactivación del virus en personas previamente contagiadas.

\section{Referencias bibliográficas}

Díaz-Castrillón (2020). SARS-CoV-2/COVID-19: The virus, the disease and the pandemic. Editora Médica Colombiana S.A. Recuperado de: https://docs.bvsalud.org/biblioref/2020/05/1096519/covid-19.pdf

Fatehi, E. (2021). Reinfection, recurrence, or delayed presentation of COVID-19? Case series and review of the literature. Journal of Infection and Public Health Volume 14, Issue 4, April 2021, Pages 474-477. https://doi.org/10.1016/j.jiph.2021.01.002

Guanche Garcell H., Barbán Arias R. (2021). Reinfección, inmunidad y prevención de la transmisión en la COVID-19. Rev haban cienc méd [Internet] [citado]; 20(4): e4101. Disponible en: http://www.revhabanera.sld.cu/ index.php/rhab/article/view/4101

Gousseff, M. (2020). Clinical recurrences of COVID-19 symptoms after recovery: Viral relapse, reinfection or inflammatory rebound? Journal of Infection, Vol 81, Issue 5 , 816-846. https://www.sciencedirect.com/science/article/pii/S0163445320304540

Overbaugh, J. (2020). Understanding protection from SARS-CoV-2 by studying reinfection. Nat Med. 26:1680. https://www.nature.com/articles/s41591-0201121-z 
Romera, I. (2021). ReinfecciónporSARS-CoV-2. Medicina Intensiva 45375-380. Disponible en: https://www.medintensiva.org/es-pdf-S0210569121000115

Salao, E (2020). Reinfección de COVID-19: Respuesta inmune y posibles causas. RECIMUNDO. https://www.recimundo.com/index.php/es/article/view/929/1496

Tillett, R. (2020). Genomic evidence for reinfection with SARS-CoV-2: A case study. Lancet Infect

Dis. https://linkinghub.elsevier.com/retrieve/pii/S1473309920307647.

To, K. (2021). Coronavirus Disease 2019 (COVID-19) Re-infection by a Phylogenetically Distinct Severe Acute Respiratory Syndrome Coronavirus 2 Strain Confirmed by Whole Genome Sequencing. Clinical Infectious Diseases, Vol 73, Issue, 2946-e2951. https://doi.org/10.1093/cid/ciaa1275

Viera, I. A. (2020). Recurrencia de infecciones de COVID-19. Rev Fac Cs Méd UNR. Vol. 1: pp. 79 a 85. http://rephip.unr.edu.ar/bitstream/handle/2133/20989/23Texto\%20del\%20art\%c3\%adculo-19-2-1020210609.pdf? sequence $=1 \&$ isAllowed $=\mathrm{y}$

Ye G., Pan Z., Pan Y. (2020). Clinical characteristics of severe acute respiratory syndrome coronavirus 2 reactivation. J Infect. 80(5): e14-e17. https://pubmed.ncbi.nlm.nih.gov/32171867/

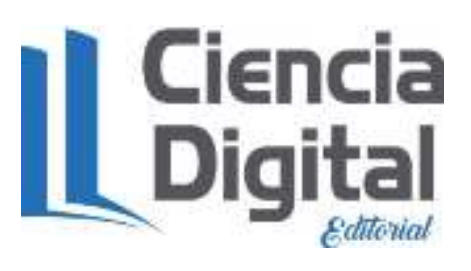




\section{PARA CITAR EL ARTÍCULO INDEXADO.}

Bermeo González, J. E., Salamea Montenegro, T. A., Delgado Larrea, M. E., Montalván Zambrano, S. E., \& Sánchez Solórzano, J. S. (2021). Recurrencia de infección por COVID-19 en población general inmunizada . Anatomía Digital, 4(4), 141-149. https://doi.org/10.33262/anatomiadigital.v4i4.1941

\section{ULiencia}

El artículo que se publica es de exclusiva responsabilidad de los autores y no necesariamente reflejan el pensamiento de la Revista Anatomía Digital.

El artículo queda en propiedad de la revista y, por tanto, su publicación parcial y/o total en otro medio tiene que ser autorizado por el director de la Revista Anatomía Digital.
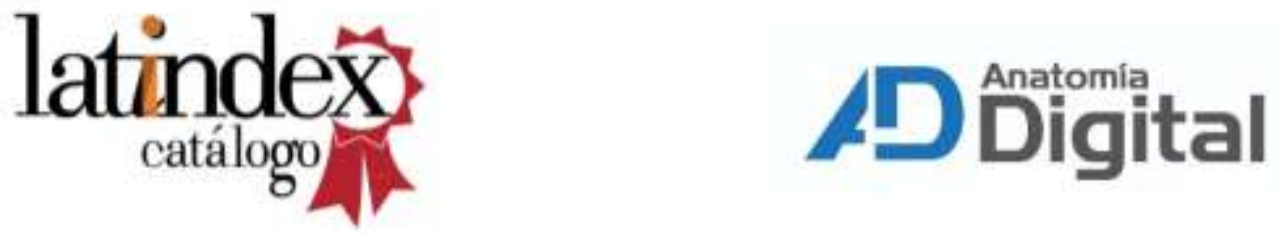